\title{
IN-SITU MULTI-MODE SENSING WITH EMBEDDED PIEZOELECTRIC WAFER ACTIVE SENSORS FOR CRITICAL PIPELINE HEALTH MONITORING.
}

\author{
Lingyu Yu, PhD \\ Mechanical Engineering Department, University of \\ South Carolina \\ Columbia, SC 29208, yu3@engr.sc.edu \\ Yuh Chao, Professor \\ Mechanical Engineering Department, University of \\ South Carolina \\ Columbia, SC 29208, chao@engr.sc.edu
}

\author{
Victor Giurgiutiu, Professor \\ Mechanical Engineering Department, University of \\ South Carolina \\ Columbia, SC 29208, giurgiut@engr.sc.edu \\ Patrick Pollock, Undergraduate student \\ Mechanical Engineering Department, University of \\ South Carolina \\ Columbia, SC 29208, pollocpj@engr.sc.edu
}

\begin{abstract}
Pipelines are important infrastructures in petroleum and gas industries which are vital to the national economy. They are typically subjected to corrosion inside of the pipe and there is an urgent need for the development of a cost-effective, nonexcavating, in-service, permanent critical pipeline damage detection and prediction system. In this paper, we proposed an in-situ multiple mode pipeline monitoring system by utilizing permanently installed piezoelectric wafer active sensors (PWAS). As an active sensing device, PWAS can be bonded to the structure or inserted into a composite structure, operated in propagating wave mode or electromechanical impedance mode. The small size and low cost (about $\sim \$ 10$ each) make it a potential and unique technology for in-situ application. Additionally, PWAS transducers can operate at a temperature as high as $260^{\circ} \mathrm{C}$ which is sufficient for most critical pipeline systems used in gas/petroleum industry. This system can be used during in-service period, recording and monitoring the changes, such as cracks, impedance, wall thickness, etc., of the pipelines over time. Having the real-time data available, maintenance strategies based on these data can then be developed to ensure a safe and less expensive operation of the pipeline systems.
\end{abstract}

The paper will first give an intensive literature review of current pipeline corrosion detection. Then, the basic principles of applying PWAS to in-situ SHM using in-plane propagation waves and impedance measurement for damage detection are studied and developed. Next, experiments were conducted to verify the corrosion detection and thickness measurement ability of PWAS sensor network in a laboratory setting and in water pipe with flowing fluid inside as well. In addition, the potential of PWAS application for high temperature pipeline thickness monitoring was also investigated.

\section{INTRODUCTION}

Pipelines are important civil infrastructures in nuclear, chemical, petroleum, and gas industries. These pipes are utilized to transport water, gas, petroleum, solvent or mixtures often operated at high temperature and subjected to vibration and harsh environment. Moreover, they are typically subjected to corrosion along the inside of the pipe. Over time, the corrosion makes the pipe wall thinner and leaves the pipes vulnerable to failure. Failure of pipes and tubular equipment in petroleum plants tend to be catastrophic, resulting in costly property damage, loss of business continuity and even fatalities. There is an ever-growing global awareness of this issue. Consequently, pipe failures have caused several governments to take actions in mandating inspection requirements. For example, due to recent failures, the US government has been increasing pipeline inspection and safety requirements, presenting new challenges to operators and inspection service companies. In the North Sea, the Norwegian government mandates that all Coiled Tubing strings be inspected before every job. With pipe integrity at the top of the agenda, owners and operators of these systems are cautious regarding the reliability and safety to avoid leaks and catastrophic failures.

\subsection{Conventional ultrasonic techniques}

Currently, there are two categories of commercially available solutions, either internal or external inspection. Internal inspection of tubes has been traditionally accomplished using a rotating transducer to scan the tube wall point by point. For instance, Krautkramer NDT has a range of standard systems for tube and pipe inspection [1]. Using robots to perform the 
inspection has also been devised [2], but their cost and complexity are high. External inspection of piping systems can be accomplished by a series of point tests performed from the outside of the pipe. If insulation covers the pipe, as it is often the case, access to the outside surface of the pipe requires removal of the insulation to perform the test, and then reinstalling the insulation after the tests are complete.

Ultrasonic techniques also permit quick and reliable measurement of thickness without requiring access to both sides of a part. The measurement is usually done by coupling the sound energy into the test piece through a water column generated by a bubbler or squirter, or in a water bath. For example, Figure 1 shows the 37DL PLUS ultrasonic thickness gage by Parametrics-NDT which is an advanced NDT ultrasonic thickness gage for applications involving pipes, tanks, and other metal structures subject to internal corrosion or erosion [3]. The operational temperature is however limited to between $-10^{\circ} \mathrm{C}$ and $50^{\circ} \mathrm{C}$. In addition, the physical size of the probe and the associated instrumentation make these devices very difficult for in-situ on-line structural health monitoring. Measurements at elevated temperatures (higher than $50^{\circ} \mathrm{C}$ ) present special challenges requiring special high temperature couplants [4]. The ultrasonic couplants will quickly vaporize if used on surfaces hotter than approximately $100^{\circ} \mathrm{C}$ [5]. Generally speaking, the main drawbacks of conventional ultrasonic inspection techniques are they are point-wise inspection methods, labor-intensive, requiring highly trained operators, and are nearly impossible to use for in-situ hightemperature SHM. More efficient methods for pipe and tube inspections using guide-wave technology have been recently developed and are discussed in the next section.

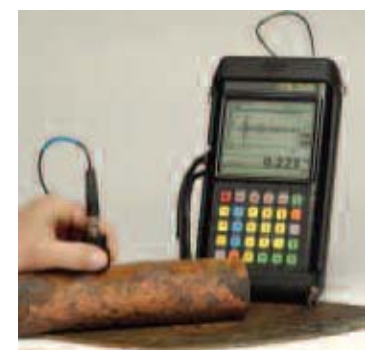

Figure 1 handheld 37DL PLUS ultrasonic thickness gage by Parametrics-NDT [3].

\subsection{Guided wave inspection}

Considering the limitations of conventional ultrasonic transducers, which leave many pipeline operators in constant search for new and improved inspection tools, a complete solution is yet to be put forward, reinforcing the emerging need for new inventions and discoveries [6]. It would be ideal if insitu pipeline monitoring and/or wall thickness sensors can be in place to record and monitor the change of wall thickness at critical locations over time.

Guided-waves (e.g. Lamb waves in thin plates) offer significant advantages over conventional ultrasonic devices [7] [9]. The use of guided ultrasonic waves for the inspection of pipes and tubes can greatly improve inspection efficiency [10]. Using guided waves, a probe can be applied to the pipe at fixed location, and then a length of the pipe can be inspected from that location. The insulation is only removed at the location where the probe is applied. Special probes are available for guided-wave pipe inspection. For example, Figure 2 shows a "comb" array probe, which surrounds the pipe for inspection.

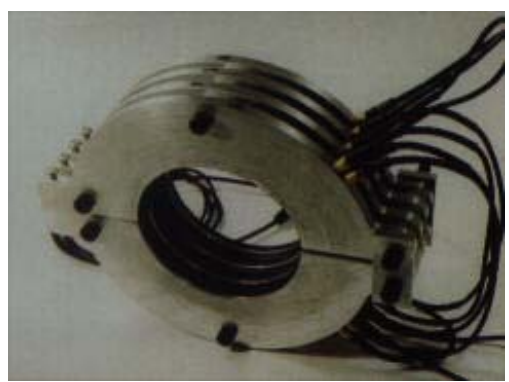

Figure 2 Comb array transducer for guided-wave pipe inspection [1]

Rose et al. utilized longitudinal guided-wave tube modes to detect circumferential crack in nuclear steam generator tubes [10]. The authors also proposed a complex-shape ultrasonic transducer that could be used to inspect a bundle of steam generator tubes using the guided wave technique. Alleyne et al. used guided waves to detect crack and corrosion in steel pipes [11]. A recent practical implementation of guided-wave inspection of pipes is provided by WaveMaker equipment and software [12]. Chahbaz, et. al. attempted to use Lamb waves to detect the corrosion in aluminum structures [13]. Application of this method to the detection of hidden corrosion in aircraft wing skins was reported by Chahbaz et al. [14]. They indicated that certain guided Lamb wave modes can transmit through the corroded area resulting in lower amplitude and longer time of flight than those when passing through an undamaged area. Alleyne et al. used guided waves to detect corrosion in steel pipes with mode conversion properties [11].

Guided waves can also be used for the thickness measurement of a vessel [15]. Commonly used conventional ultrasonic transducers for guided wave generation are often not suitable because of potential couplant contamination. The noncontact electromagnetic acoustic transducer (EMAT) offers advantages over conventional transducers. The measurements can be made very quickly and without need for liquid couplant [5]. A schematics is shown in Figure 3 for a non-contact EMAT apparatus. The temperature limitations of the EMAT system are $0^{\circ} \mathrm{C}$ to $60^{\circ} \mathrm{C}$ in continuous contact and up to $80^{\circ} \mathrm{C}$ in intermittent contact [16]. The highest allowable temperature is dependent upon the temperature of the permanent magnet and the electrical component in each specific EMAT design. However, limitations of the magnetostrictive EMAT transducers exist, including: (1) the potential for magnetic saturation from the permanent magnet; (2) automated measuring using EMAT requires probe holders made of stainless steel (Figure 3) and holders must be adjusted for optimal signal response during each calibration or setup [17]; (3) the minimum measurable wall thickness and measurement accuracy are not nearly as good as what can be achieved normally with a conventional ultrasonic transducer. 


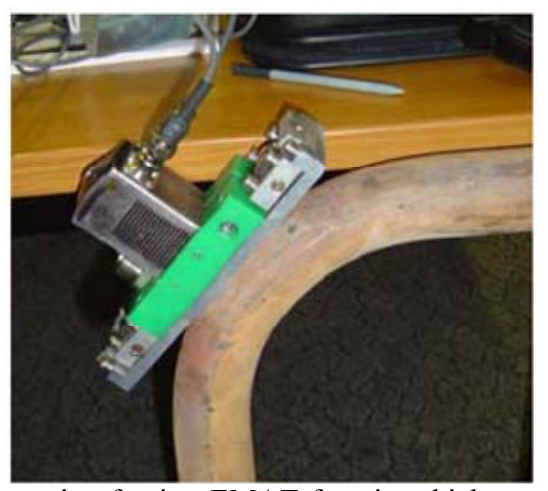

Figure 3 Schematic of using EMAT for pipe thickness measurement [16] [17]

In summary, various ultrasonic based devices are laborintensive and need highly trained operators. Conventional ultrasonic transducers will tolerate temperatures up to only $50^{\circ}$ C. Higher temperature measurement can be done when special couplants were used. However, it requires special care and effort in working with the couplants. All these devices are bulky, expensive and need extra mechanisms for operation. Considering the high temperature, thick insulation, and harsh environment (e.g. vibration and weather) under which pipelines are operated, the physical size of the probe, couplants and associated instrument/accessories of ultrasound based device make it impossible for in-situ, real-time measurement of pipe thickness for pipeline structural health monitoring (SHM).

This paper presents an in-situ multi-mode diagnostics/monitoring of critical pipeline system utilizing embedded piezoelectric wafer active sensors (PWAS) guided waves. PWAS can be used as active sensing device, providing the bidirectional energy transduction from the electronics into the structure, and from the structure back into the electronics. The small size and low cost (about $\sim \$ 10$ each) make PWAS a potential and unique technology for in-situ application. PWAS can be bonded to the structure or inserted into a composite structure, operated in propagating wave mode or electromechanical impedance mode. Additionally, PWAS transducers can operate at a temperature as high as $260^{\circ} \mathrm{C}$ which is sufficient for many critical pipeline systems used in gas/petroleum industry. As active sensors, PWAS can be used as either transmitter or receiver and connected to data concentrators and wireless communicators as both transmitters and receivers to generate or receive guided waves [18]. PWAS can also be used as collocated electro-mechanical (E/M) impedance sensor-actuators that permit effective modal identification in a wide frequency band. In a pipeline sensing system, the PWAS can be embedded into the pipes by mounting them directly on the outside surface of a pipe, under the protective shielding and insulation, and left in place to perform their structural health-monitoring task (Such thing would be unthinkable with conventional ultrasonic transducers, which are bulky, obtrusive, and expensive).

\section{IN-SITU PIPELINE HEALTH MONITORING WITH PIEZOELECTRIC WAFER ACTIVE SENSORS}

\subsection{PWAS guided waves transducers}

The modeling and understanding of the interaction between surface-mounted piezoelectric-wafer active sensors and structures is essential for the successful development of this method. This effort is essential to the generation of highfrequency guided waves of thickness type. Though previous researchers have separately modeled guided waves in thin-wall structures, and while others have modeled piezoelectric-wafer active sensors, none has captured the intimate interaction between these surface-mounted strain transducers and the structure in the thickness mode as applied to a pipe/tube.

\subsubsection{PWAS principles}

The general constitutive equations of linear piezoelectric materials given by ANSI/IEEE Standard 176-1987, describe a tensorial relation between mechanical and electrical variables (mechanical strain, $S_{i j}$, mechanical stress, $T_{k l}$, electrical field, $E_{k}$, and electrical displacement $D_{j}$ ) in the form

$$
\begin{aligned}
& S_{i j}=s_{i j k l}^{E} T_{k l}+d_{k i j} E_{k} \\
& D_{j}=d_{j k l} T_{k l}+\varepsilon_{j k}^{T} E_{k},
\end{aligned}
$$

where $s_{i j k l}^{E}$ is the mechanical compliance of the material measured at zero electric field $(E=0), \varepsilon_{j k}^{T}$ is the dielectric permittivity measured at zero mechanical stress $(T=0)$, and $d_{k i j}$ is the piezoelectric coupling between the electrical and mechanical variables.

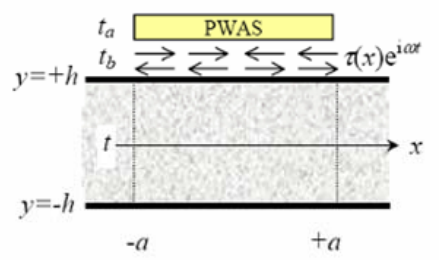

Figure 4 Interaction between the PWAS and the structure

As conventional ultrasonic transducers, PWAS can be used with propagation guided waves in pitch-catch or pulse-echo mode (as illustrated in Figure 5).

\subsubsection{PWAS Lamb wave tuning}

For Lamb waves, there are at least two Lamb modes, A0 and S0, existing simultaneously for many SHM approaches, where the product of the wave frequency and structure thickness falls in the range of $0 \sim 1 \mathrm{MHz} \cdot \mathrm{mm}$. Within this range, A0 mode is sensitive to damage associated with disbands and corrosion while S0 mode is sensitive to crack damage. The tuning of Lamb wave attempts to modify the excitation parameters such as to excite certain mode for detecting certain damage. With wedge-coupled conventional ultrasonic transducers, guided wave tuning is performed by varying the frequency and the wedge angle until a maximum response is recorded. The change in frequency modifies the wave speed of the dispersive 
guided wave, while the change of wedge angle modifies the wave conversion relationship in Snell's law. Certain combinations of wedge-angles and excitation frequencies were able to generate increase response in certain guided-wave modes [10].

PWAS are capable of geometric tuning through matching between their characteristic direction and the half wavelength of the excited Lamb mode. In this application, PWAS tuning means to tune the excitation at a frequency where S0 Lamb mode dominates and A0 Lamb mode is minimized. Giurgiutiu and Bottai showed that given the PWAS length $2 a$, plate thickness $2 d$, and material properties $\mu$ and $\lambda$, it is possible to find frequencies at which only one mode is excited (tuning frequency) [19]. A plane-strain analysis of the PWAS-structure interaction using the space-domain Fourier analysis has been developed to illustrate the principle of PWAS Lamb wave mode tuning. That is to say, a selected Lamb mode can be tuned by choosing the appropriate frequency and PWAS dimensions. A plot of the strain solution in the $0 \sim 700 \mathrm{kHz}$ bandwidth for an aluminum plate of $1 \mathrm{~mm}$ thickness installed with $7 \mathrm{~mm}$ round PWAS is presented in Figure 6a while Figure $6 \mathrm{~b}$ gives the group velocity. In Figure 6a, a S0 tuning frequency around $300 \mathrm{kHz}$ can be found, where the amplitude of the A0 mode is minimized while that of the S0 mode is still strong. Therefore, we have tuning of the S0 mode and are able to reject the A0 mode. These predictions have been extensively verified experimentally [19]. We see that Lamb wave tuning offers considerable advantages, allowing us to select Lamb waves that are most appropriate for the particular application being considered.

(a)
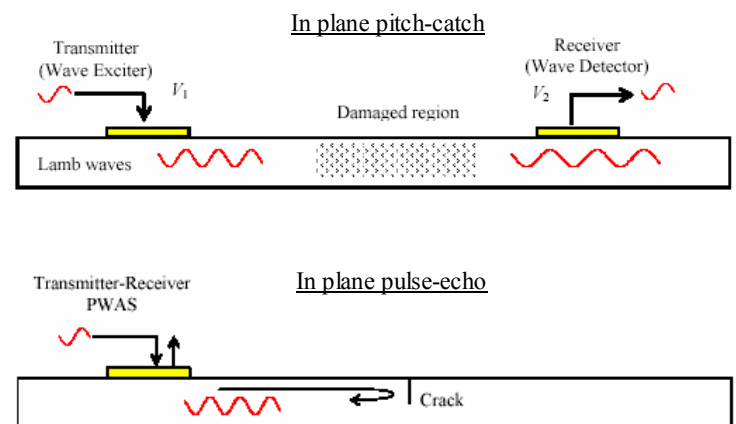

Figure 5 guided-wave applications: (a) pitch-catch method in plane motion; (b) pulse-echo method in plane motion
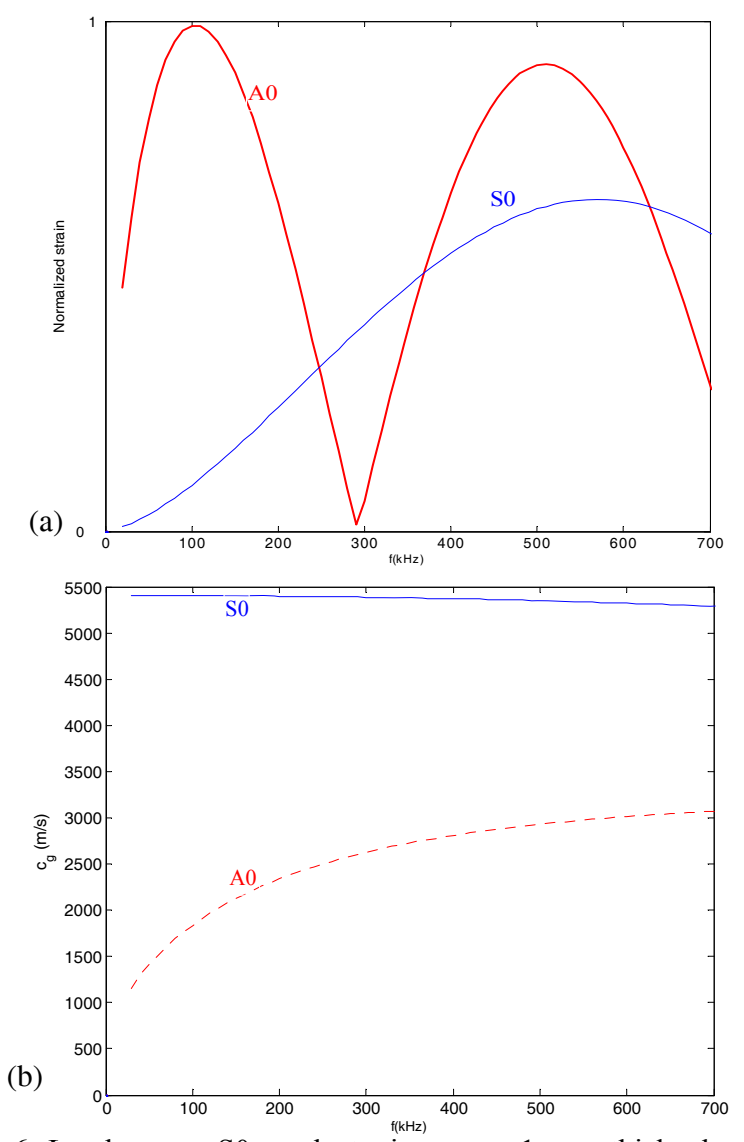

Figure 6 Lamb wave S0 mode tuning on a $1-\mathrm{mm}$ thick aluminum plate using 7-mm round PWAS within $0 \sim 700 \mathrm{kHz}$ range. (a) strain curve; (b) group velocity (dispersion curves)

\subsection{PWAS impedance measurement}

The principles of electro-mechanical (E/M) impedance method are illustrated in Figure 7. The drive-point impedance presented by the structure to the active sensor can be expressed as the frequency dependent variable $Z_{\mathrm{str}}(\omega)=k_{\mathrm{str}}(\omega) / j \omega=k_{e}(\omega)$ $\omega_{\mathrm{m}}^{2}(\omega)+j \omega c_{e}(\omega)$. Through the mechanical coupling between PWAS and the host structure, on one hand, and through the E/M transduction inside the PWAS, on the other hand, the drive-point structural impedance is reflected directly in the electrical impedance, $Z(\omega)$, at the PWAS terminals

$$
Z(\omega)=\left[j \omega c\left(1-\kappa_{31}^{2} \frac{\chi(\omega)}{1+\chi(\omega)}\right)\right]^{-1}
$$

where $C$ is the zero-load capacitance of the PWAS and $\kappa_{31}$ is the E/M cross coupling coefficient of the PWAS $\left(\kappa_{31}=d_{31} / \sqrt{\bar{s}_{11} \bar{\varepsilon}_{33}}\right)$, and $\chi(\omega)=k_{s t r} / k_{P W A S}$ with $k_{P W A S}$ being the static stiffness of the PWAS. During a frequency sweep, the real part of the E/M impedance, $Z(\omega)$, follows the up and down variation as the structural impedance as it goes through the peaks and valleys of the structural resonances and antiresonances [20]. The E/M impedance method is applied by scanning a predetermined frequency range in the high $\mathrm{kHz}$ band (up to $10 \mathrm{MHz}$ ) and recording the complex impedance spectrum. By comparing the real part of the impedance spectra 
taken at various times during the service life of a structure, meaningful information can be extracted pertinent to structural degradation and ongoing damage development.

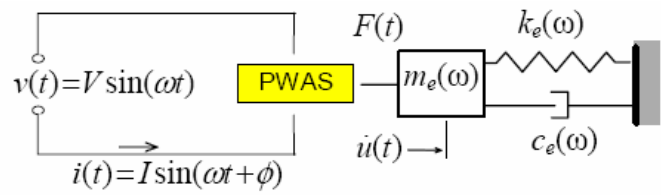

Figure 7 E/M coupling between PWAS and structure [20]

\subsection{PWAS thickness measurement}

Like conventional ultrasonic transducer (Figure 8a), PWAS can also generate pressure wave for thickness measurement. Pressure waves can be excited by PWAS transducers at a single surface which propagate in thickness direction. When the waves pulse encounters the other surface, backscattered reflections are created, sent back, and received at the origin in the form of an echo (Figure 8b). The time-difference between the pulse and the echo is proportional to twice the distance between the transmitter and the boundary, and serves as the basis for thickness measurement.

(a)

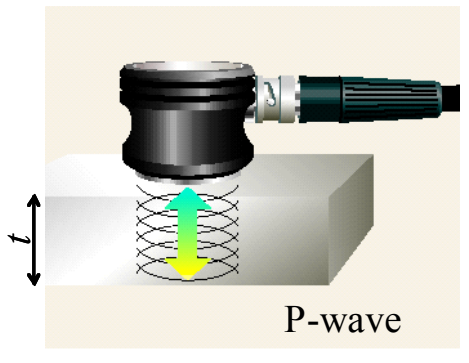

(b)

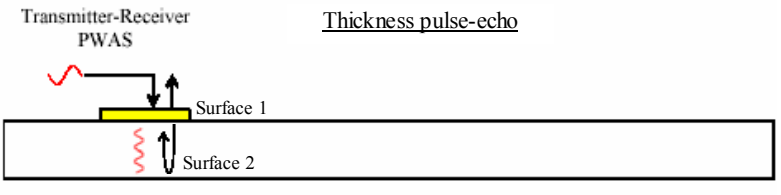

Figure 8 Thickness measurement with pressure wave. conventional ultrasonic transducer; (b) PWAS

\section{PWAS MULTI-MODE SENSING SYSTEM FOR CORROSION DETECTION}

Guided Lamb waves have opened new opportunities for cost effective detection of damage in structures. The use of Lamb waves to detect the corrosion in aluminum structures using the pitch-catch method has been explored by Chahbaz et al [13] [14]. Alleyne et al detected corrosion in pipes using the pulseecho method using the mode conversion method [7]. Zhu and Rose conducted experimental study on hidden corrosion detection by using the ultrasonic guided waves combined with BEM numerical simulation [21].

The proposed PWAS multi-mode sensing system will take advantage of PWAS ability: (1) to serve as guided wave transducer, (2) to measure structural impedance spectrum, and (3) to directly measure the wall thickness. Once a PWAS sensing system, i.e., PWAS sensor network, is established on the structure, it will detect the corrosion defect in the structure by using the three methods simultaneously by altering driving frequencies. A typical such system is illustrated in Figure 9. The detection procedure consists of three steps:

1. Lamb waves inspection using pitch catch method. The data will be analyzed by a damage index method. The pitch-catch pair which is suffering the largest change will be considered as having corrosion on that path.

2. Once the path is determined, E/M impedance spectrum will be obtained for all the PWAS along the path and analyzed by damage index method. The transducer which has the largest change will be determined as the one on the corrosion.

3. Finally, direct thickness measurements will be conducted to quantify the thinning.

By using this strategy, the corrosion area will be located and measured.

\subsection{Corrosion diagnosis-damage index analysis}

In using pitch-catch method for guided wave damage detection, if the wave travels through a region where there is a change in material properties, such as thickness decreasing in corrosion, the directly transmitted signal will be modified and the receiving signal will provide good information about the corrosion development across the transmitting and receiving transducer path. The change can be obtained by subtracting the baseline data recorded for the structure without corrosion from the receiving data for the structure with the developing corrosion damage. The difference is a good indicator for the corrosion detection parameter since it carries information of both amplitude changes and the phased changes from the corrosion growth. The damage index (DI) is defined as the relative ratio of the difference between each measurement and baseline signals to the baseline signal

$$
\text { Damage index }(\mathrm{DI})=\sqrt{\frac{\sum_{j=0}^{N-1}\left[s_{i}(j)-s_{0}(j)\right]^{2}}{\sum_{j=0}^{N-1} s_{0}^{2}(j)}}
$$

where $s_{i}$ is the $i^{\text {th }}$ measurement and $s_{0}$ is the baseline signal, and $N$ is the data length.

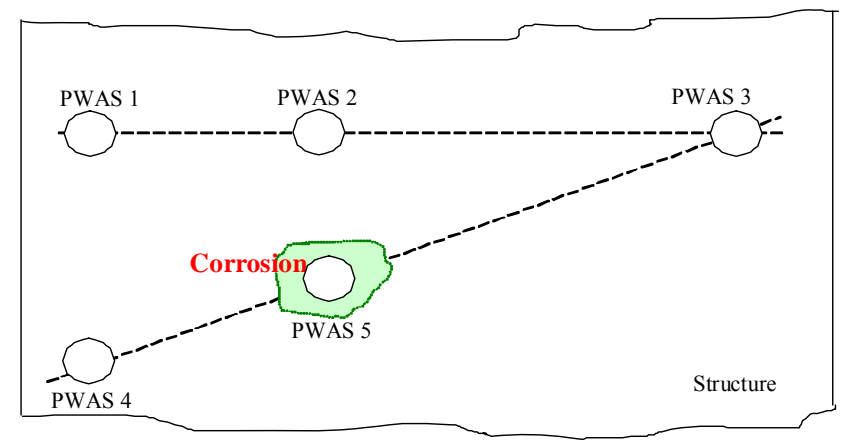

Figure 9 PWAS multi-mode corrosion detection system 


\subsection{Plate thickness measurement}

A proof-of-concept investigation was performed to verify that PWAS can indeed be used for plate thickness measurement. Figure 10a shows a schematic of the setup and Figure $10 \mathrm{~b}$ is the actual experimental setup. The system consists of a plate specimen with a square or round PWAS attached to one surface of the plate. The PWAS are connected with thin insulated wires to a signal bus that ends at a digital pulse/echo device. Under electric excitation, the PWAS generates an elastic Lamb wave pulse that spreads out into the plate following a thickness wave pattern. When the wave pulse hits the other surface, it is reflected back as an echo to the PWAS. The elastic Lamb waves are transmitted back as an electric signal that is then picked up by the digital oscilloscope synchronized with the pulse/echo device. This setup is equivalent to the measurement of the time of flight of the reflective echo for the calculation of plate thickness. A typical pulse-echo signal is shown in Figure 11 , which indicates a delay of $\Delta t$ between the initial pulse and the first reflected echo. The echo strength is strongest at first, which is the first reflected wave from the lower surface, and becomes weaker for the later echoes. Given the wave speed $c$ of the specified material, the thickness $T$ can be calculated as

$$
T=\frac{\Delta t \cdot c}{2}
$$

To verify the proposed thickness measurement method, we performed a series of laboratory experiments using the setup of Figure 10 at room temperature. Both aluminum and steel plates are considered and the results are presented in Table 1. As shown in Table 1, except for the thinner aluminum plate with $3.3 \mathrm{~mm}$ thickness, the other two measurements yield excellent results.

(a)
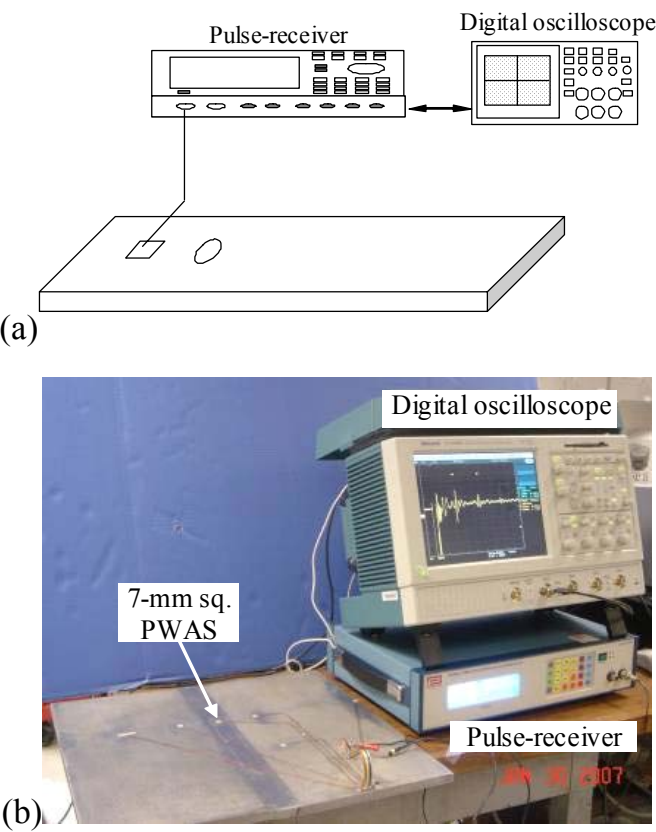

Figure 10 Lamb-wave PWAS thickness measurement experiment. (a) schematic of experimental setup; (b) practical laboratory experiment setup

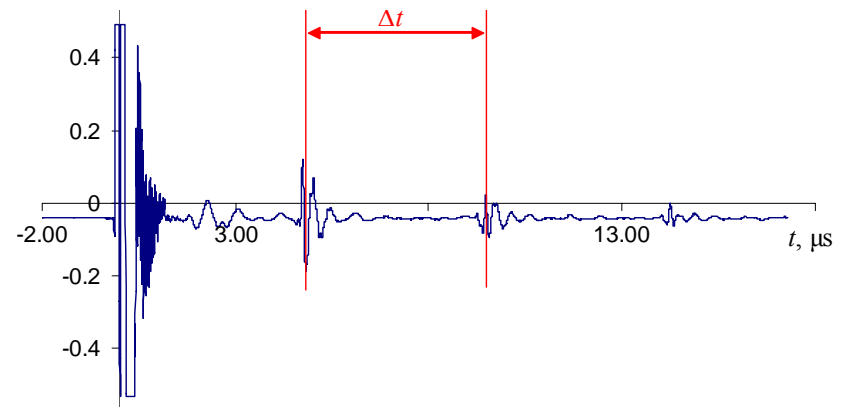

Figure $11 \mathrm{~A}$ pulse-echo signal captured by a $7-\mathrm{mm}$ round PWAS attached to a $13.5-\mathrm{mm}$ thick steel plate under room temperature

Table 1 Metallic specimens specifications and measurements

\begin{tabular}{|c|c|c|c|c|c|}
\hline Material & $\begin{array}{c}T_{0} \\
(\mathrm{~mm})\end{array}$ & $\begin{array}{c}c \\
(\mathrm{~mm} / \mu \mathrm{s})\end{array}$ & $\begin{array}{c}\Delta t \\
(\mu \mathrm{s})\end{array}$ & $\begin{array}{c}T_{i} \\
(\mathrm{~mm})\end{array}$ & $\begin{array}{c}\text { Error } \\
(\%)\end{array}$ \\
\hline $\begin{array}{c}\text { Aluminum } \\
\text { 2024 T4 }\end{array}$ & 3.3 & 6.37 & 0.842 & 2.68 & $18.8 \%$ \\
\hline $\begin{array}{c}\text { Aluminum } \\
\text { 2024 T4 }\end{array}$ & 6.4 & 6.37 & 2.05 & 6.529 & $2.5 \%$ \\
\hline Steel 4340 & 13.5 & 5.85 & 4.59 & 13.43 & $0.5 \%$ \\
\hline
\end{tabular}

$T_{0}$-theoretical thickness; $T_{i}$-measured thickness

\subsection{PWAS corrosion detection on plate specimen}

The proposed multi-mode sensing has been tested on a $500 \mathrm{~mm}$ x $500 \mathrm{~mm} \times 3.229 \mathrm{~mm}$ rectangular aluminum plate. A five 7 $\mathrm{mm}$ round PWAS sensor network was installed as illustrated in Figure 12a. A $5 \mathrm{~mm} \times 3.8 \mathrm{~mm}$ area is used to simulate the corrosion by removing material from the plate, as shown in Figure 12b. The thickness change is listed in Table 2

(a)

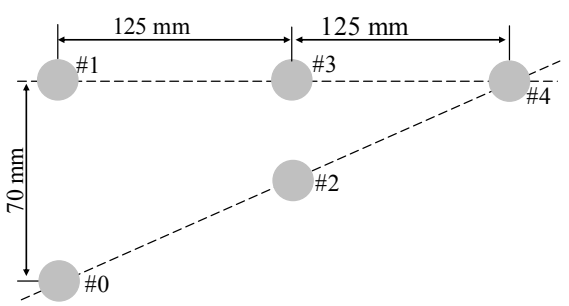

(b)

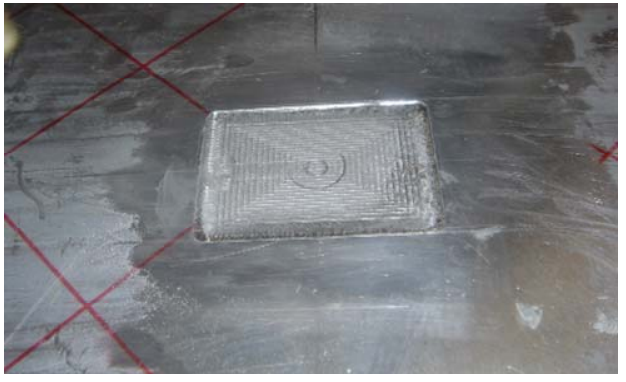

Figure 12 Corrosion detection on an aluminum plate. (a) a 5-PWAS network; (b) simulated corrosion

Table 2 Corrosion simulation on aluminum plate 


\begin{tabular}{|c|c|c|}
\hline Records & $\Delta d_{i}(\mathrm{~mm})$ & $\Delta d_{i} / d_{0}(\%)$ \\
\hline 0 & 0 & 0 \\
\hline 1 & 0.381 & 11.81 \\
\hline 2 & 0.7366 & 22.81 \\
\hline 3 & 0.9398 & 29.1 \\
\hline 4 & 1.09728 & 33.98 \\
\hline 5 & 1.27 & 39.33 \\
\hline
\end{tabular}

$d_{0}$-original thickness; $\Delta d_{i}$-removed thickness

The corrosion is located exactly at the same position of PWAS \#2 on the other side of the plate. We conducted pitch-catch experiments on the PWAS pair 14 (PWAS\#1 and PWAS\#4) and pair 04 (PWAS\#0 and PWAS\#4). Using DI analysis, it was identified that the corrosion is located on the path of pair 04 . Then impedance spectra for PWAS \#0, \#2, and \#3 were recorded and compared. PWAS \#2 show the largest spectrum change and was identified as where the corrosion is. Finally, PWAS \#2 was used to measure the local plate thickness.

\subsubsection{Pitch-catch corrosion detection}

The pitch-catch experiment sends out a 3-count tone-burst signal from a HP 33120A signal generator to one PWAS and the received signal at the other PWAS is recorded by a TDS210 digital oscilloscope. Frequency tuning was first conducted to find out the "sweet spot" where S0 mode is minimized and A0 mode dominates, which was finally chosen at $57 \mathrm{kHz}$.

Signals received on PWAS \#4 when PWAS \#0 sent at various corroded depth are shown in Figure 13a while the received signals when PWAS \#1 sent are shown in Figure 13b (only the first arrival wave packets are shown). It can be noticed from Figure 13a that the first arriving A0 wave packet suffers shifting (being delayed) in time scale and the amplitude increased at first (from data set baseline 0 to record 4) and then decreased (for record 5 when thinning is about 40\%). Such difference between records can be quantified with the DI analysis as the DI curve given in Figure 14a. The reason for the DI curve first increasing and then decreasing observation needs to be further investigated theoretically and experimentally. However, it coincides with our previous research result [23].

For the data set of PWAS pair 14, since the corrosion is away from the path, the arriving wave packet is not significantly affected by the growth of corrosion as the data of pair 04 (Figure 13b). The DI curve is provided in Figure 14b.

When frequency increases, the S0 mode begins to appear. However, the presence of S0 mode will not affect the corrosion detection using A0 mode since S0 mode is not significantly modified by the growth of corrosion. A test at the frequency of $120 \mathrm{kHz}$, from PWAS \#0 to PWAS \#4, was taken and the recorded signals are present in Figure 15a. The first arriving wave pack, quite small, is the S0 mode and the second arriving wave pack, much larger, is the A0 mode. The data shows that the S0 mode wave packs did not change significantly with the corrosion development. This finding confirms our previous theoretical investigation that A0 mode is more sensitive to corrosion defect while S0 mode is more sensitive to throughthe-thickness crack by Giurgiutiu et al [24].

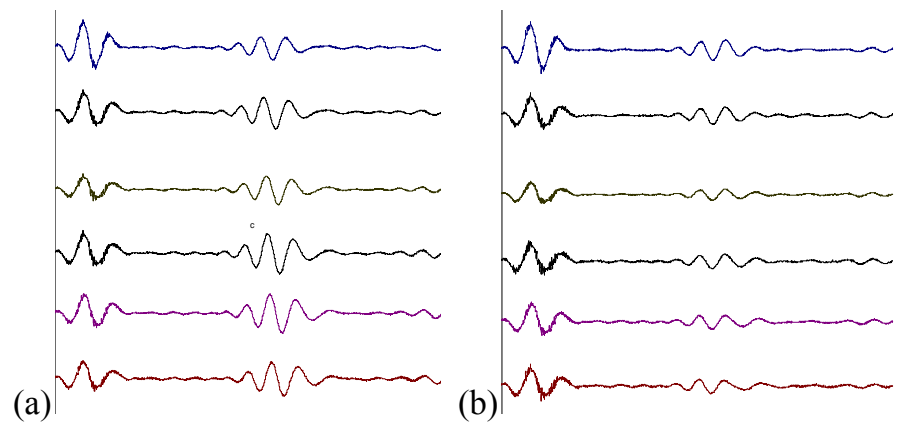

Figure 13 Pitch-catch signals on aluminum plate at various corroded depth. (a) received signals at PWAS \#4 when PWAS \#0 sent; (b) received signals at PWAS \#4 when PWAS \#1 sent

(a)

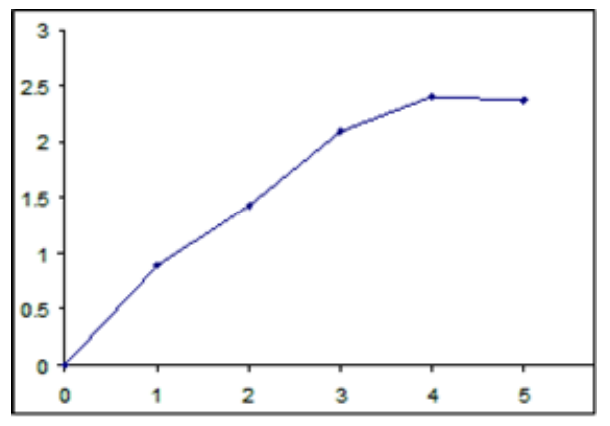

(b)

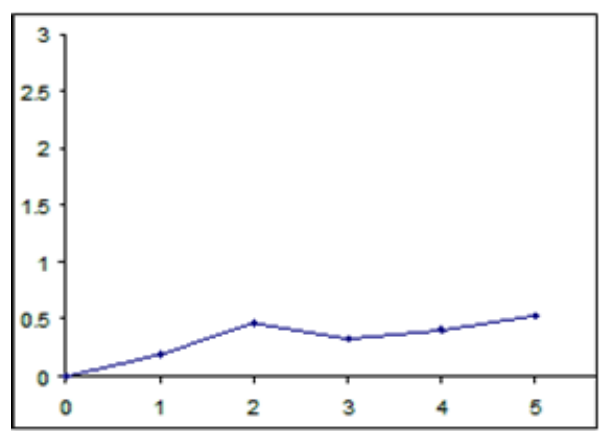

Figure 14DI analysis on pitch-catch signals for different PWAS pairs. (a) DI curve for PWAS \#4 receives while PWAS \#0 sent; (b) DI curve for PWAS \#4 receives while PWAS \#1 sent

(a)

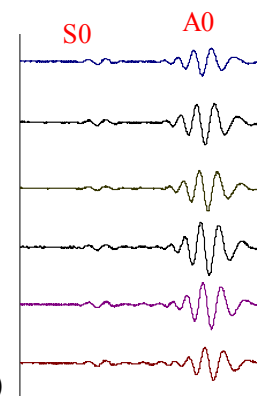

(b)

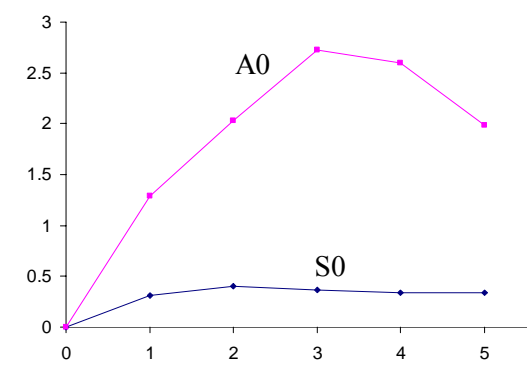

Figure 15 Indication of corrosion growth. (a) recorded signals at PWAS \#4 while PWAS \#0 sent at $120 \mathrm{kHz}$; (b) DI analysis on A0 and S0 mode packs, respectively 


\subsubsection{Impedance spectrum}

After path 0-4 was identified as the corroded path, impedance measurement was used to locate the corrosion position. Impedance spectra of PWAS \#0, \#2, and \#3 were recorded by HP 4194A impedance analyzer over the frequency band 200 2000 kHz where several fundamental modes were observed, given in Figure 16. (Note that \#4 was omitted since it is symmetric with \#0 about \#2 and \#3 was intentionally investigated in order to show the difference between \#2 and \#3)

(a)

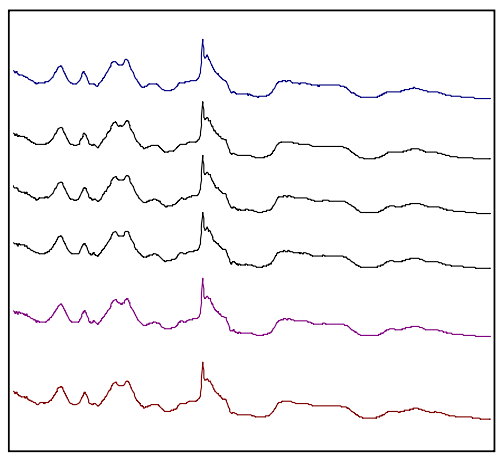

(b)
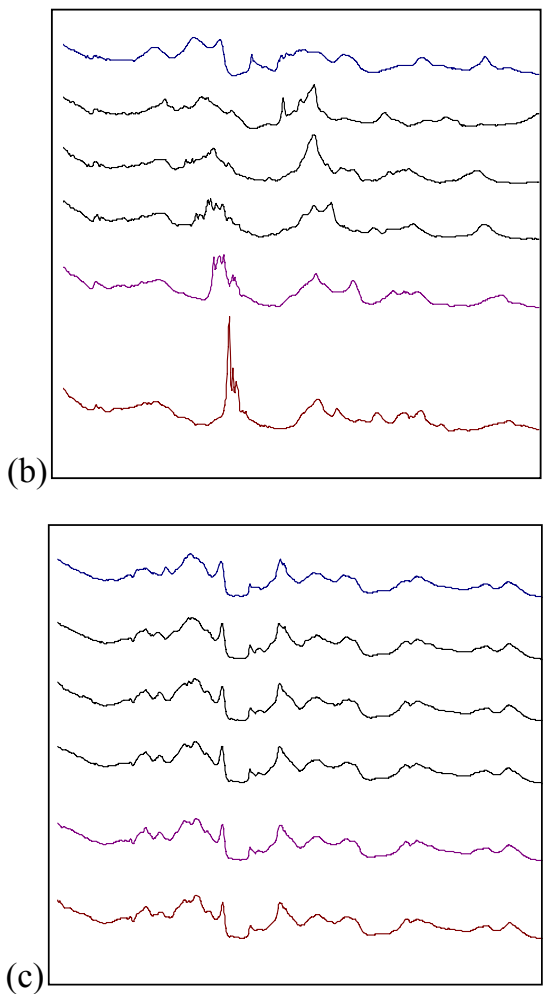

Figure 16 Recorded E/M impedance spectra at PWAS \#0, \#2, and \#3 at various corroded depth. (a) spectra of PWAS \#0; (b) spectra of PWAS \#2; (c) spectra of PWAS \#3

The spectrum difference between "baseline" data and "corroded" data can be quantified by processing the data, the real part of the E/M impedance spectra, with DI method. The DI curve of the spectrum of PWAS \#2 is shown in Figure 17.

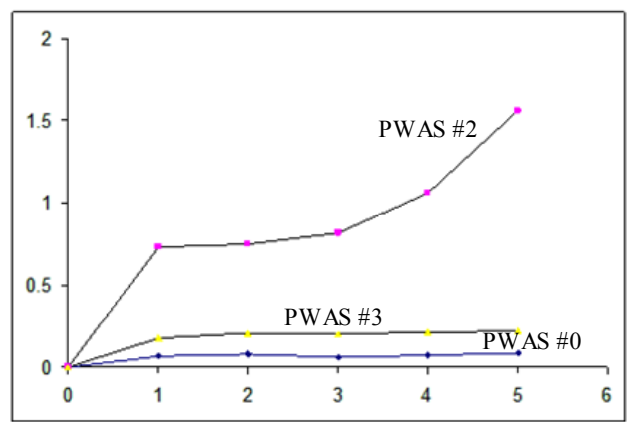

Figure 17DI curve of impedance spectrum

\subsubsection{Thickness measurement}

Once the sensor undergoing most significant impedance change was identified, i.e., PWAS \#2, it is used directly measure the thickness at its position. To compare the performance of PWAS thickness measurement, a $30 \mathrm{MHz}$ conventional ultrasonic transducer has also been used in parallel for comparison. The measurement results are given in Table 3. It shows that PWAS provides reasonable results despite their low cost. Subsequent work is needed to investigate error ranges of both conventional transducers and PWAS. A high performance thickness gauge is to be considered and further investigation on the error ranges of the two transducers is to be conducted.

Table 3 Thickness measurement using PWAS \#2 and ultrasonic transducer

\begin{tabular}{|c|c|c|c|c|c|}
\hline Records & $\begin{array}{c}T_{0}, \\
\mathrm{~mm}\end{array}$ & $\begin{array}{c}T_{\text {PWAS }}, \\
\mathrm{mm}\end{array}$ & $\begin{array}{c}\varepsilon_{\text {PWAS }}, \\
\%\end{array}$ & $\begin{array}{c}T_{\text {ultrasonic, }}, \\
\mathrm{mm}\end{array}$ & $\begin{array}{c}\varepsilon_{\text {ultrasonic }} \\
\%\end{array}$ \\
\hline 0 & 3.229 & 3.354 & 3.87 & 3.069 & 4.96 \\
\hline 1 & 2.848 & 2.9072 & 2.08 & 2.7022 & 5.12 \\
\hline 2 & 2.4924 & 2.5034 & 4.41 & 2.3481 & 5.78 \\
\hline 3 & 2.2892 & 2.298 & 3.84 & 2.174 & 5.03 \\
\hline 4 & 2.137 & 2.218 & 3.79 & 2.062 & 3.50 \\
\hline 5 & 1.959 & 2.1742 & 10.98 & 1.839 & 6.13 \\
\hline
\end{tabular}

$T_{0}$-theoretical thickness, $c$-wave velocity, $6.212 \mathrm{~mm} / \mu \mathrm{s}$

\section{ENVIROMENTAL EXPERIMENTS}

In order to make PWAS a potential technology for in-situ pipeline sensing system, environmental investigation of using PWAS has been conducted by Giurgiutiu and Lin [24]. In this paper, we are focused on the field testing and high temperature potential of PWAS transducers.

\subsection{Thickness measurement on water pipe under room temperature}

PWAS thickness measurement field test was conducted at the hydraulic lab at the civil engineering department, USC (Figure 18). The test ran from static water $(0 \mathrm{~m} / \mathrm{s})$, medium water flow $(0.161 \mathrm{~m} / \mathrm{s})$, to high water flow $(0.29 \mathrm{~m} / \mathrm{s})$. From measurements shown in Figure 19, we verified that PWAS, 7-mm round or square, work consistently at various water flow. 


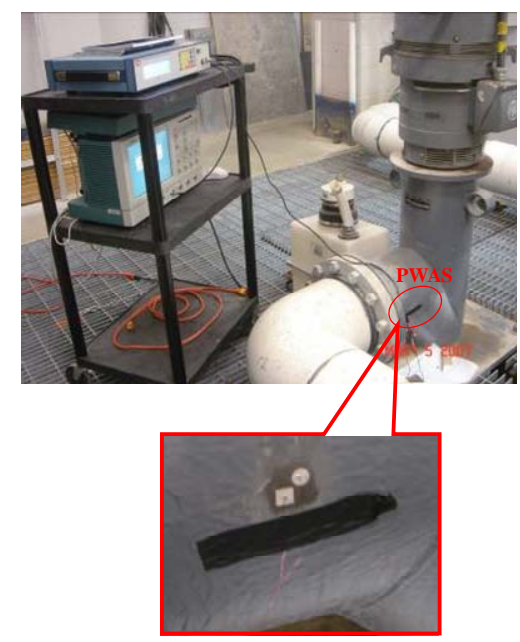

Figure 18 PWAS thickness measurement on water pipe

(a)
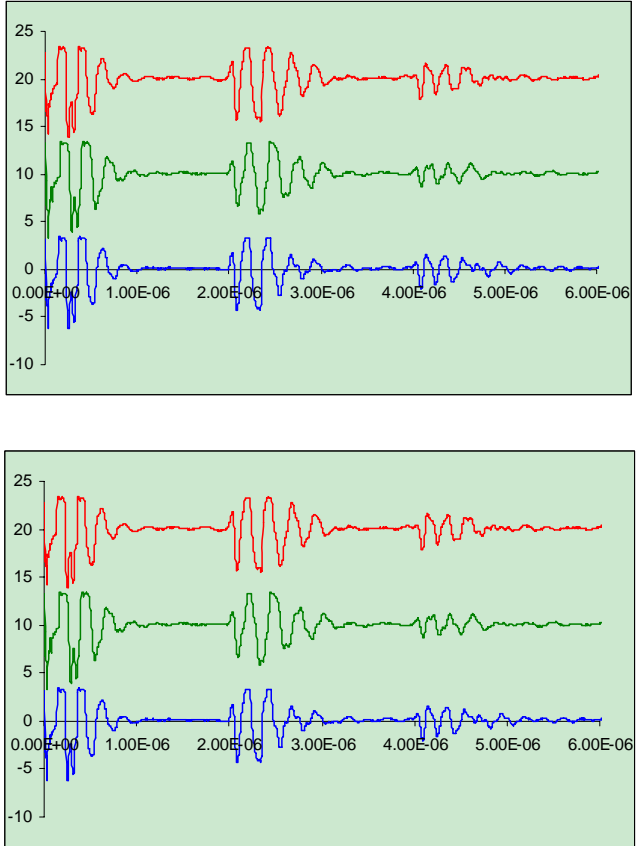

(b)

Figure 19 Thickness measurement during water pipe experiment. (a) using 7-mm round PWAS; (b) using 7-mm square PWAS

\subsection{PWAS high temperature testing}

Tolerance, stability and consistency in high temperature operation are required for PWAS to use as the proposed sensor for pipeline applications. In this regard, we have attempted to investigate the performance of PWAS at high temperatures. Figure 20 shows the PWAS impedance spectrum measured after exposures to temperatures ranging from $38^{\circ} \mathrm{C}$ to $371^{\circ} \mathrm{C}$ $\left(100^{\circ} \mathrm{F}\right.$ to $\left.700^{\circ} \mathrm{F}\right)$. It is noticed that below $260^{\circ} \mathrm{C}\left(500^{\circ} \mathrm{F}\right)$ the PWAS showed strong anti-resonant peaks, which is indicative of a strong piezoelectric response. However, between $260^{\circ} \mathrm{C}$ and $316^{\circ} \mathrm{C}\left(500^{\circ} \mathrm{F}\right.$ and $\left.600^{\circ} \mathrm{F}\right)$, the PWAS was gradually loosing the peaks indicating a loss of the piezoelectric properties. The impedance spectrums of PWAS that were exposed to temperatures of $316^{\circ} \mathrm{C}$ and $371^{\circ} \mathrm{C}\left(600^{\circ} \mathrm{F}\right.$ and $\left.700^{\circ} \mathrm{F}\right)$ were completely flat. This indicates that the maximum working temperature of PWAS cannot exceed $\sim 260^{\circ} \mathrm{C}\left(500^{\circ} \mathrm{F}\right)$, which is sufficiently high for most petrochemical and gas pipeline applications. High temperature PWAS tests inside an oven including impedance measurement, pitch-catch, and pulse-echo methods will be conducted in the future.

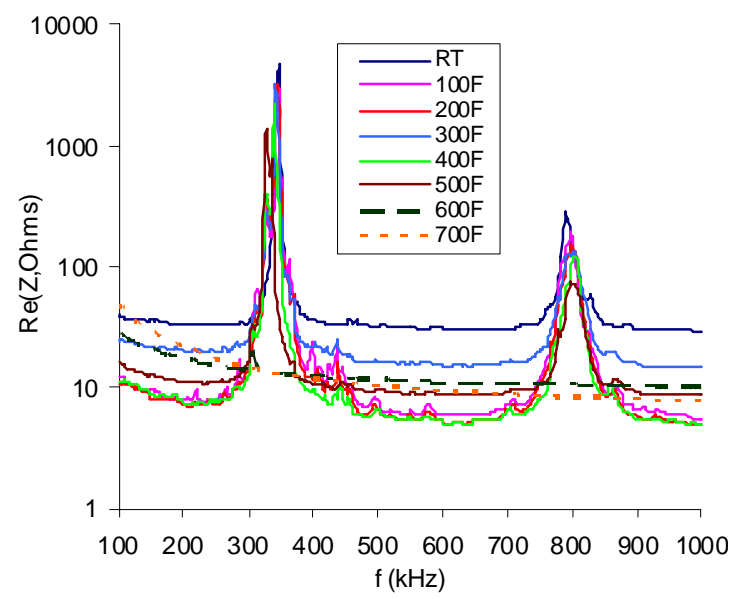

Figure 20PWAS impedance spectrum variation with temperature showing PWAS “dies" out between $260^{\circ} \mathrm{C}$ and $316^{\circ} \mathrm{C}$

\section{CONCLUSIONS}

Structural health monitoring with active sensors (active SHM) uses inexpensive and unobtrusive transducers permanently attached to the structure to interrogate it at will. The piezoelectric wafer active sensors (PWAS) used in this paper are small, inexpensive, and minimally invasive. They interrogate the structure through guided waves traveling at large distances into plate and shell structures. The ultimate objective of this research is to create the multi-mode sensing methodology and technology that can permit the installation of an in-situ structural health-monitoring network and perform both propagating wave and E/M impedance SHM in high temperature pipeline systems. Such a health-monitoring network is slated to improve the diagnostic efficiency and eliminate the regular shutdowns for preventive inspection and maintenance, thus considerably reducing the operation costs, decreasing personnel exposure, and increasing the safety of critical pipelines.

In this paper, we reviewed the state of the art of pipeline corrosion detection and proposed the multi-mode sensing using PWAS transducer. Preliminary findings on pipeline inspection leveraging on prior experience with health monitoring of plate structures using PWAS were presented. Then, a brief introduction of the principle of PWAS, Lamb wave, and PWAS in-situ health monitoring, was given. A detailed description of the proposed method follows with extensive laboratory experiments to verify the corrosion detection ability for plate specimen at room temperature. The multiple sensing uses the pitch-catch method to find out the sensor path where corrosion is occurring and impedance spectrum to locate the sensor closest to the corrosion. Damage index (DI) diagnosis method was used to quantify the change in pitch-catch and impedance 
measurements. It is noticed that when the plate is suffering thinning, the DI curve increases at first while when the thickness decreases to about $40 \%$ of the original thickness, the curve increased instead. Due to the localization property of impedance spectrum, the DI curve of the impedance spectra shows that only the sensor undergoing corrosion changes significantly while the sensor near the corrosion does not change that much. After the corrosion position is found, the current thickness is measured to determine the exact change. The thickness measurement using PWAS transducers shows good consistence and the performance is comparable to the conventional ultrasonic transducer. Investigations into field test on water pipe and high temperature testing were also provided in the end. The PWAS thickness measurement is not affected by the flowing water inside the pipe. The highest temperature for the healthy performance of PWAS was found to be $260^{\circ} \mathrm{C}$ which is suitable for most gas pipeline.

Currently, the effect of using different types of PWAS (e.g., square or round PWAS, decreased size PWAS) for the accuracy of the measured results is underway. The dependence of changes in signals on the material, geometry (e.g. curved or flat surface), and environment will be further investigated both theoretically and experimentally. As founded in this paper, S0 mode wave will not be affected by the corrosion defect while A0 mode changes significantly with corrosion development. Experiments on using different modes to detect different defects will be conducted to further investigate this potential of embedded PWAS guided waves monitoring. The proposed PWAS sensing network is going to be used on actual metallic pipes. In addition, new hardware design is underway for a "packaged PWAS" sensor such that a directed, focused plane wave front can be generated to reduce the reduction in signal and enhance the accuracy of the measurement.

\section{ACKNOWLEDGMENTS}

This material is based upon work supported by the National Science Foundation under Grant \#CMS-0408578 and \#CMS0528873 and by the Air Force Office of Scientific Research under Grant \# FA9550-04-0085.

\section{REFERENCES}

1 Krautkramer NDT (1998) "Emerging Technology - Guided Wave Ultrasonics", NDTnet, Vol. 3, No. 6, June 1998

2 Harker, A. H. (1988) Elastic Waves in Solids, British Gas, UK, 1988

3 Envirocoustics S.A. (2007) http://www.envirocoustics.gr/products/ultrasonic/ut thick_37dlpl us eng.htm

4 Fowler , K.A.; Elfbaum , G.M.; Smith, K.A.; Nelligan, T.J. (1997) "Theory and application of precision ultrasonic thickness gaging", NDTnet, October 1997, Vol.2 No.10

5 Olympus (2006) http://www.olympusndt.com/en/ndtapplication/87-id.209715235.html

6 Jamoussi, A. (2005) "Robotic NDE: A New Solution for In-line Pipe Inspection", $3^{\text {rd }}$ MENDT - Middle East Nondestructive Testing Conference \& Exhibition - 27-30 Nov 2005 Bahrain, Manama

7 Alleyne, D. N.; Cawley P. (1992) “Optimization of Lamb Wave Inspection Techniques", NDT_E International, Vol. 25 (1992), No. 1, pp. 11-22
8 J.L Rose, "Recent Advances in Guided Wave NDE," 1995 IEEE Ultrasonics Symposium Proceedings, (Piscataway, NJ: IEEE, 1995), pp. 76101-770.

9 J.L. Rose, "A Baseline and Vision of Ultrasonic Guided Wave Inspection Potential," ASME J. Pressure Vessel Technology: Special Issue on Nondestructive Characterization of Structural Materials, 124 (3) (August 2002), pp. 273-282

10 Rose, J. L.; Ditri, J. J.; Pilarski, A.; Rajana, K.; Carr, F.T. (1994) "A guided wave inspection technique for nuclear steam generator tubing", NDT\&E International, Vol. 27, pp. 307-330, 1994

11 Alleyne, D.N., Pavlakovic, B., Lowe, M.J.S., Cawley, P. (2001) "Rapid, Long Range Inspection of Chemical Plant Pipework Using Guided Waves," Review of Progress in QNDE, Vol. 20, (2001), pp. 180-187

12 Guided Ultrasonics Ltd. (2003) http://www.guidedultrasonics.com/index.html

13 Chahbaz, A.; Mustafa, V.; Hay, D. R. (1996) Corrosion Detection in Aircraft Structures using Guided Lamb Waves, ASNT Fall Conference 1996, NDTet, Vol. 1, No. 11, November 1996, http://www.ndt.net/article/tektrend/tektrend.htm

14 Chahbaz A., Gauthier J.; Brassard, M.; Hay, D. R. (1999) " Ultrasonic Technique for Hidden Corrosion Detection in Aircraft Wing Skin"; Proceedings of the $3^{\text {rd }}$ Joint Conference on Aging Aircraft, 1999

15 Luo, W. and Rose, J.L. (2003) "Guided wave thickness measurement with EMATs", Insight-Non-Destructive Testing and Condition Monitoring, Volume: 45, Issue: 11, Page(s): 735739

16 Lee, K. and Nelligan, T. (2004) "The Use of Magnetostrictive EMAT Transducers on Oxide Scaled Boiler Tubes" http://www.ndt.net/article/wcndt2004/pdf/noncontact_ultrasonics $/ 308$ lee.pdf

17 Starmans electronics s.r.o. http://www.olympusndt.com/en/ndt-application/87id.209715208.html

18 Giurgiutiu, V. (2006) "In-situ Structural Health Monitoring, diagnostics and Prognostics System Utilizing Thin Piezoelectric Sensors", U.S. patent, Patent No. US 7,024,315 B2, April 4, 2006

19 Bottai, G., Giurgiutiu, V. (2005), "Simulation of the Lamb Wave Interaction between Piezoelectric Wafer Active Sensors and Host Structure", Proceedings of SPIE Vol.5765, Smart Structures and Materials 2005: Sensors and Smart Structures Technologies for Civil, Mechanical, and Aerospace Systems, pp. 259-270

20 Giurgiutiu, V. (2003) "Embedded Ultrasonics NDE with Piezoelectric Wafer Active Sensors", Journal Instrumentation, Mesure, Metrologie, Lavoisier Pub., Paris, France, RS series 12M, Vol. 3, No. 3-4, 2003, pp. 149-180

21 Zhu, W.; Rose, J.L.; Agarwala, V.S. (1999) "Experimental Study on Hidden Corrosion/Delamination Detection with Ultrasonic Guided Waves", Defense Technical Information Center, Accession \# ADD344128

22 Giurgiutiu, V.; Bao, J.; Zhao, W. (2003) "Piezoelectric-Wafer Active-Sensor Embedded Ultrasonics in Beams and Plates", Experimental Mechanics, Sage Pub., Vol. 43, No. 4, December 2003, pp. 428-449

23 Thomas, D. T.; Welter, J. T.; Giurgiutiu, V. (2004) “Corrosion Damage Detection with Piezoelectric Wafer Active Sensors", $2^{\text {nd }}$ European Workshop on Structural Health Monitoring, July 7-9, 2004, Munich, Germany, pp. 1253-1261

24 Giurgiutiu, V.; LIN, B. (2004) "Durability and Survivability of Piezoelectric Wafer Active Sensors for Structural Health Monitoring using the Electromechanical Impedance Technique", Proceedings of the 2004 ASME International Mechanical Engineering Congress, November 13-19, 2004, Anaheim, CA, paper \# IMECE2004-60974 